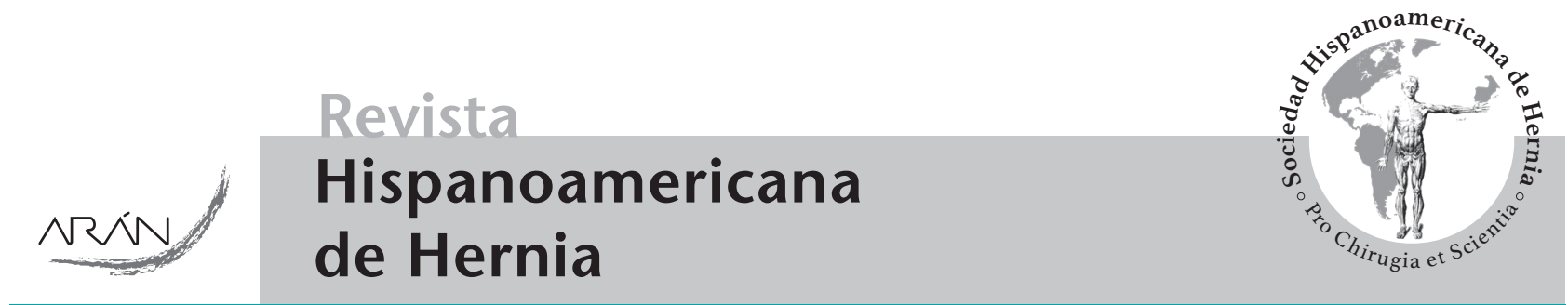

www.grupoaran.com

Nota clínica

\title{
Apendicitis aguda del anciano dentro de una hernia de Petit. Reporte de un caso
}

Recibido: 07-11-2017

Aceptado: 10-06-2018

Palabras clave:

Hernia lumbar, apendicitis, tratamiento, malla.

\section{Resumen}

Introducción: Las hernias lumbares representan el $2 \%$ del total de hernias ventrales. La hernia de Petit pertenece al grupo de hernias lumbares congénitas ocupando el $10 \%$ de estas. El defecto herniario es a nivel del triángulo lumbar inferior, limitado por la cresta ilíaca como base, el músculo oblicuo externo como borde lateral y el músculo dorsal ancho como borde medial. No existen reportes de la presencia de una apendicitis aguda dentro de una hernia de este tipo. Presentamos el caso de un paciente con apendicitis aguda en una hernia de Petit.

Caso clínico: Hombre de 80 años de edad que inicia con cuadro de dolor abdominal persistente, inespecífico y difuso de 1 mes de evolución, acompañado de hipertermia no cuantificada, anorexia, dificultad para canalizar gases y estreñimiento. En la exploración física se encontró distención abdominal moderada, ruidos intestinales disminuidos, rebote positivo y resistencia muscular franca en todo abdomen. Se observo abultamiento en región lumbar derecha de 5 $\times 5 \mathrm{~cm}$, duro y no reductible, muy doloroso a la palpación. Se estableció el diagnóstico hernia lumbar estrangulada y se programó exploración quirúrgica y hernioplastia lumbar, encontrando apéndice cecal con datos de inflamación aguda, abscedada y con plastrón de epiplón dentro de un saco herniario a través de un defecto herniario de Petit. Presentó además peritonitis purulenta generalizada, decidiendo realizar laparotomía exploradora para revisión y lavado del resto de la cavidad, apendicectomía y cierre primario del defecto aponeurótico por la contaminación. El estudio histopatológico mostró apendicitis aguda perforada (grado IV). Evolución favorable a un año del posoperatorio sin recidiva.

Discusión: La apendicitis aguda asociada a una hernia de Petit resulta un diagnóstico excepcional.
Key words:

Lumbar hernia, appendicitis, treatment, mesh.

\begin{abstract}
Introduction: Lumbar hernias represent $2 \%$ of the total ventral hernias. Petit's hernia belongs to the group of congenital lumbar hernias occupying $10 \%$ of them. The hernia defect is at the level of the lower lumbar triangle, limited by the iliac crest as the base, the external oblique muscle as the lateral border and the broad dorsal muscle as the medial border. There are no reports of the presence of acute appendicitis within a hernia of this type. We present the case of a patient with acute appendicitis in a Petit hernia.

Case report: Male, 80 years old. He started with persistent, non-specific and diffuse abdominal pain with an evolution of a month, accompanied by non-quantified hyperthermia, anorexia, difficulty in channeling gas and constipation. At the physical examination, he revealed moderate abdominal distension, decreased bowel sounds, positive rebound, and frank muscular resistance throughout the abdomen. A $5 \times 5 \mathrm{~cm}$ lump was observed. It was hard and not reducible; very painful at palpation. The diagnosis of a strangulated lumbar hernia was established and surgical exploration and lumbar hernioplasty were scheduled, finding the appendix with an acute inflammation, abscess and an omentum plastron within a hernia sac through a small hernia defect. He also presented generalized purulent peritonitis, deciding to perform an exploratory laparotomy to check and wash the rest of the cavity, appendectomy and primary closure of the aponeurotic defect due to contamination. The histopathological study showed an acute perforated appendicitis (grade IV). After a year it was observed a favorable evolution without a recurrence.

Discussion: An acute appendicitis associated with a Petit's hernia is an exceptional diagnosis.
\end{abstract}

* Autor para correspondencia: Ana de Montserrat Medina Sifuentes. Unidad Médica de Alta Especialidad UMAE T1 IMSS. Bajío. Roble 509, Fraccionamiento Las Arboledas. 20020 Aguascalientes. Ags. (México)

Correo electrónico: cirugiamedinasifuentes@gmail.com 


\section{Introducción}

Las hernias lumbares de Petit corresponden a las llamadas «hernias raras», denominadas así por su baja incidencia de presentación y a las que eventualmente el cirujano se enfrenta. Las hernias lumbares representan el $2 \%$ del total de hernias ventrales ${ }^{1}$ y se clasifican en congénitas, traumáticas, incisionales y espontáneas ${ }^{2,3}$. La hernia de Petit y la de Grynfeltt pertenecen al grupo de hernias lumbares congénitas que ocupan apenas el $10 \%$ de estas. La hernia de Petit se da a nivel del triángulo lumbar inferior, limitado por la cresta del hueso ilíaco como base, el músculo oblicuo externo como borde lateral y el músculo dorsal ancho como borde medial. El riesgo de incarceración de una hernia lumbar en general es del $25 \%$ y de estrangulación de un 8 a un $18 \%{ }^{1}$.

El tratamiento de las hernias lumbares en la actualidad es a través de una hernioplastia con el uso de una prótesis de malla, ya sea por abordaje abierto o laparoscópico. Debido al tamaño por lo general pequeño de las hernias congénitas, sus resultados en cuanto a recidivas son mejores en este tipo de hernias que los obtenidos en las hernias incisionales, traumáticas o las que presentan un componente de denervación muscular ${ }^{4}$.

La presencia del apéndice cecal dentro del saco herniario de una hernia de Petit, con o sin proceso agudo, no ha sido descrito en la literatura médica hasta el momento. Presentamos el caso de un paciente anciano con cuadro apendicular agudo perforado, dentro de un saco herniario de una hernia de Petit, simulando una hernia lumbar estrangulada, en un hospital rural, prácticamente sin recursos diagnósticos y con mínimos insumos quirúrgicos.

\section{Caso clínico}

Se trata de un paciente masculino de 80 años de edad con antecedente de hernia inguinal derecha hace 10 años manejada mediante hernioplastia inguinal con malla. Negó otras patologías previas. Inició su cuadro con dolor abdominal persistente, inespecífico y difuso de 1 mes de evolución, acompañado de hipertermias no cuantificadas, anorexia, dificultad para canalizar gases y estreñimiento, sin recibir algún tratamiento formal para este cuadro. Ante la agudización del dolor abdominal se presentó en el Servicio de Urgencias del Hospital Rural de Ario de Rosales Mich, México. A la exploración física se encontró paciente con mucosas deshidratadas y taquicárdico $110 \times$ '. Abdomen presentando distensión moderada, rebote positivo, resistencia muscular franca generalizada y ruidos peristálticos disminuidos. Se detectó un abultamiento en región lumbar derecha de $5 \times 5 \mathrm{~cm}$, duro, no reductible y muy doloroso a la palpación. En Servicio de Urgencias se establece el diagnóstico probable de hernia lumbar estrangulada y ante la falta de recursos de imagenología en el turno nocturno, se decide exploración quirúrgica con hernioplastia lumbar.

\section{Técnica quirúrgica}

Bajo anestesia local y con el paciente en posición de lumbotomía derecha, se incidió en forma transversal en región lumbar derecha sobre el sitio del saco herniario. Se localizó el saco herniario que mostró edema y datos inflamatorios agudos por presencia de proceso agudo en su interior (fig. 1). Se disecó en su totalidad hasta la base del anillo herniario, el cual fue de un diámetro de $3 \times 3 \mathrm{~cm}$ (fig. 2). Al efectuar la apertura del saco herniario se obtuvo abundante cantidad de material purulento, apreciando presencia de este material incluso dentro de la cavidad peritoneal. Se encuentra plastrón de epiplón cubriendo al apéndice cecal necrosado y perforado sin lograr identificar adecuadamente su base en el ciego. Se decidió no seguir el procedimiento por esta vía ante la gran contaminación de la cavidad abdominal y la dificultad de hacerlo a través de un orificio herniario muy pequeño. Se introdujo el apéndice en la cavidad abdominal para realizar la apendicectomía, se exploró el resto de la cavidad y se realizó un lavado peritoneal a través de una laparotomía media. Se cerró el defecto herniario con puntos simples de Prolene del 0 afrontando los bordes sin utilización de material protésico debido a la contaminación severa. Se lavó la herida de lumbotomía y se cerró por planos. Se colocó al paciente en decúbito ventral y se efectuó laparotomía por línea media. Se revisa cavidad abdominal sin encontrar más patología que el apéndice perforado (fig. 3) y una peritonitis generalizada secundaria. El ciego se apreció muy laxo y casi sin fijación a pared sobre línea de Toldt. Se realizó apendicectomía convencional y lavado exhaustivo de cavidad, dejando drenajes por el contrabertura, dando por terminado el procedimiento.

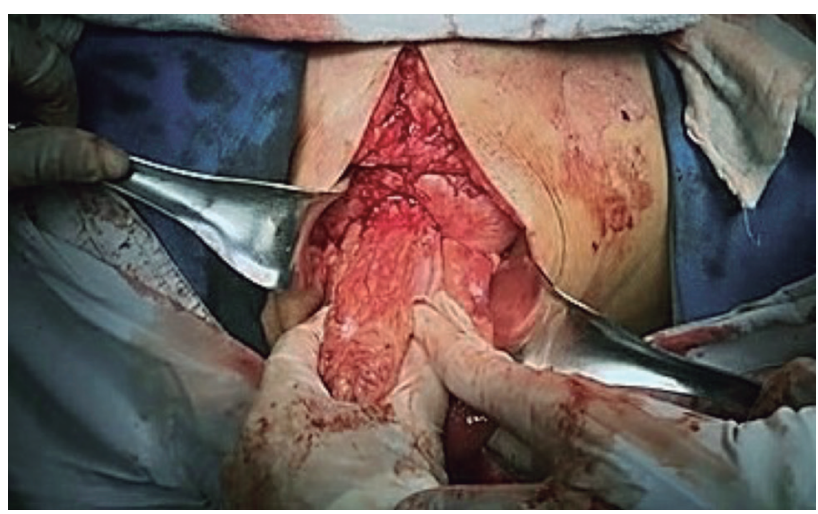

Figura 1. Presencia del saco herniario de la hernia lumbar con contenido de proceso apendicular agudo.

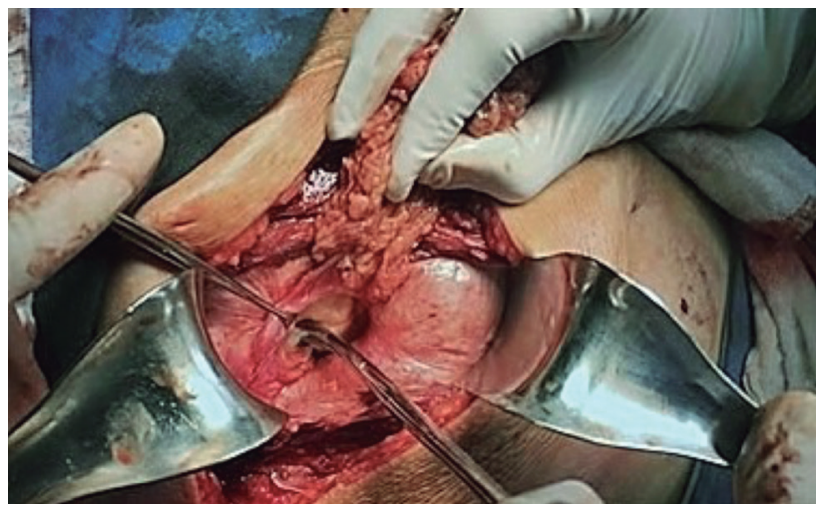

Figura 2. Hernia de Petit a nivel del triángulo lumbar inferior, limitado por la cresta del hueso ilíaco como base, el músculo oblicuo externo como borde lateral y el músculo dorsal ancho como borde medial. El defecto herniario de $3 \times 3 \mathrm{~cm}$, una vez reducido el contenido del saco herniario. 


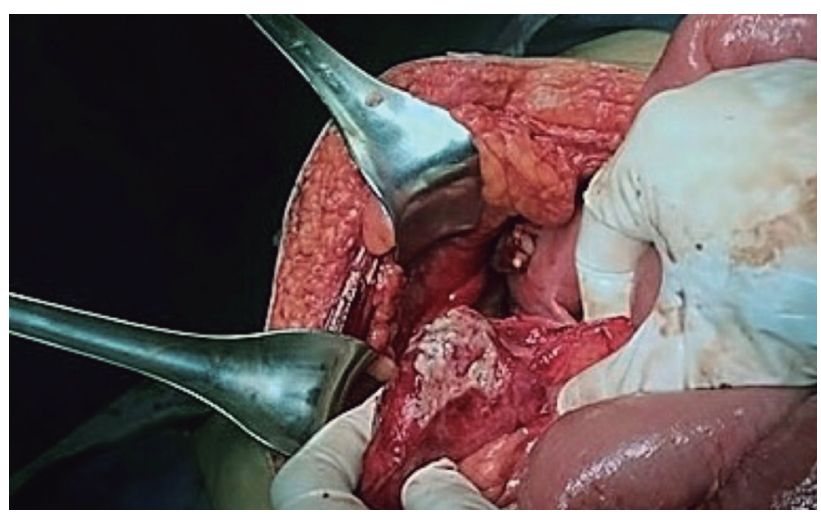

Figura 3. Abordaje por laparotomía en línea media para lograr exposición adecuada del colon derecho, ciego y apéndice. Se observa el proceso apendicular con perforación y necrosis del cuerpo en su porción media.

La evolución posoperatoria intrahospitalaria cursa sin eventualidades y fue dado de alta al quinto día de la cirugía, tolerando vía oral y sin datos de proceso infeccioso a ningún nivel (heridas o abscesos residuales). El estudio histopatológico reportó apendicitis aguda perforada (grado V). El paciente presenta un seguimiento de 12 meses hasta el momento, sin manifestaciones clínica de recidiva herniaria

\section{Discusión}

La región lumbar presenta 2 zonas de debilidad anatómica, delimitadas en un triángulo superior y en otro inferior, ambos compuestos por dos lados musculares y uno de estructura ósea (arco costal el superior o cresa ilíaca el inferior) ${ }^{5,6}$. La descripción de los límites anatómicos del triángulo inferior fue realizada por Petit en 1783, y la descripción del triángulo superior, por Grynfelt en $1866^{2}$, por lo que cada una de estas hernias lleva su nombre, respectivamente. Se considera que estas hernias son de origen congénito, aunque pueden manifestarse en cualquier momento a lo largo de la vida del paciente, desde la niñez, o hacerse manifiesta hasta la edad de adulto mayor como en el caso que presentamos. La frecuencia de presentación de estos tipos de hernia congénita es de apenas un $10 \%$ de todas las hernias lumbares ${ }^{1}$.

Este tipo de hernias por lo general tienen un defecto herniario con diámetro de pequeño a moderado, sin embargo, los sacos herniarios pueden alcanzar dimensiones importantes dependiendo del tiempo de evolución de la hernia. Aunque en su gran mayoría las hernias lumbares producen escasa sintomatología al paciente mientras son reductibles, en un $25 \%$ de los casos estas hernias se incarceran, dando mayor sintomatología, principalmente dolor ${ }^{1,7,8}$. El contenido de los sacos de estas hernias puede ser grasa retroperitoneal o colon ascendente por su cercanía a los defectos. Ocasionalmente en hernias con defectos grandes, el contenido será de asas de intestino delgado. Es poco factible que otras estructuras anatómicas tengan posibilidad de introducirse en el defecto debido a la localización tan posterior de los defectos herniarios de Petit y Grynfeltt. Aun así, en el caso que reportamos la localización del apéndice cecal dentro del saco herniario de la hernia de Petit fue favorecida por la movilidad exagerada y falta de fijación habitual del ciego y presencia de un apéndice largo.

De un 8 a $18 \%$ de las hernias lumbares presentará eventualmente un cuadro agudo de estrangulación, lo que constituye una verdadera urgencia quirúrgica como lo han reportado en sus casos publicados diferentes autores como Orozco y Skrekas ${ }^{7,8}$. Resulta excepcional encontrar un cuadro agudo en una hernia lumbar por la presencia del apéndice cecal con proceso agudo simulando una hernia estrangulada como en el presente caso reportado.

Aunque el diagnóstico de una hernia es clínico y debe estar basado en la anamnesis y en la exploración física, es recomendable la complementación diagnóstica en casos electivos con estudios de imagen específicos como la tomografía axial computarizada $^{8}$. Solo en casos de hernias lumbares traumáticas, espontáneas e incisionales en las que se piense que existe un componente de denervación, se pensará en realizar una electromiografía.

El abordaje para la reparación de las hernias lumbares debe individualizarse, siendo igual de efectivos los abordajes abiertos y laparoscópicos con uso de prótesis de malla para las hernias de tipo congénito y la mayoría de las incisionales. Moreno ${ }^{2,4,5}$ recomienda el abordaje abierto cuando exista la presencia de denervación muscular al requerir de preferencia una técnica doble para la colocación de la malla (técnica de sándwich) y realizar plicatura muscular en los casos de sobredistensión del complejo muscular lateral. En los casos de hernias estranguladas con contaminación franca o como en nuestro caso, ocasionado por una apendicitis aguda perforada, se recomienda manejar la urgencia con reducción del contenido si hay adecuada vitalidad o resección del segmento isquémico y el defecto herniario será reparado sin la colocación de un cuerpo extraño como la malla, aún con el riesgo más elevado de recidiva a mediano y largo plazo de la hernia.

\section{Conclusiones}

La apendicitis aguda asociada dentro de una hernia lumbar de Petit resulta un diagnóstico excepcional. El cuadro clínico clásico de apendicitis puede verse modificado por esta topografía anormal, facilitando la progresión natural de la enfermedad.

\section{Bibliografía}

1. Mayagoitia GJC, Cisneros MHA, Álvarez QR, Bernal GR, Guillen JM. Manejo de las hernias lumbares de la pared abdominal. Seguimiento a mediano y largo plazo de sus resultados. Cir Gral. 2008;30(4):197-203.

2. Moreno A, Baena EG, Calle MC, Martínez JAT, Albasini JLA. Controversies in the current management of lumbar hernias. Arch Surg. 2007; 142:82-8.

3. Mayagoitia GJC. Acceso anterior para hernioplastia lumbar psincisional. Cir Gral. 2001;23(1):40-3.

4. Moreno A, Torralba J, Morales G. Open vs. laparoscopic repair of secondary lumbar hernias. Surg Endosc. 2005;19:184-7.

5. Moreno A, Morales Conde S, Barreiro Morandeira F, Hernández Granados P, Feliu Palú X. Hernias lumbares. Hernias de Spiegel. Hernia obturatriz; Guías clínicas de la Asociación Española de Cirujanos. 2. ${ }^{a}$ ed. Cirugía de la pared abdominal, pp. 241-252.

6. Moreno-Egea A. Hernia lumbar, espacio oval y reconstrucción de la pared abdominal posterior. Rev Hispanoam Hernia. 2017;5(3):95-9.

7. Orozco Gil N, Martinez Ballester M, Bañuls Matoses A, Montesinos Meliá C, Caro Pérez F. Hernia de Petit incarcerada: a propósito de un caso. Rev Hispanoam Hernia. 2016;04:33-6.

8. Skrekas G, Stafyla VK, Papalois VE. A Grynfeltt hernia: Report of a case. Hernia. 2005;9:188-91.

9. Armstrong A O, Hamel B, Grignon J M, Ndoye O, Hamel R. Lumbar hernia: Anatomical basis and clinical aspects. Surg Radiol Anat. 2008;30:533-7. 